\title{
DIAGNOSIS OF VORTEX INDUCED VIBRATION OF A GRAVITY DAMPER
}

\author{
Lorenzo SCAPPATICCI ${ }^{1}$, Francesco CASTELLANI ${ }^{2}$, Davide ASTOLFI ${ }^{2}$, Alberto GARINEI ${ }^{1}$ \\ ${ }^{1}$ Guglielmo Marconi University, Department of Sustainability Engineering, via Plinio 44,00193, Roma, Italy, \\ 1.scappaticci@unimarconi.it, a.garinei@unimarconi.it \\ ${ }^{2}$ University of Perugia, Department of Engineering, Via Duranti, 06125, Perugia, Italy, \\ davide.astolfi@unipg.it, francesco.castellani@unipg.it
}

\begin{abstract}
A gravity damper is a one-way valve, employed for regulating the airflow rate in ducts, generally constituted by a series of rectangular panels (closure sections), connected to an articulated quadrilateral synchronizing the movements. If the device needs to process large masses of high speed air, as common in the case of energy conversion systems, disadvantageous dynamic effects can occur. In this study, vortexinduced vibration (VIV), occurring on a gravity damper for high values of the Reynolds number, is investigated. The analysis of this work couples numerical methods (Computational Fluid Dynamics with Large-Eddy Simulation turbulence model and Finite Element Method) to experiments: a full-scale accelerometric measurement campaign is actually performed at the wind tunnel facilities of the University of Perugia. VIVs are diagnosed and quantified through the experimental vibration analysis, which is interpreted through numerical simulations. The large amplitude of VIV is interpreted as due to a tendency towards lockin because of the approaching of the vortex shedding frequency to a natural vibration mode of the system. The integrated numerical and experimental framework finally inspires two different design solutions for mitigating the amplitude of VIV: these strategies are tested at the wind tunnel and they are indeed shown to be effective.
\end{abstract}

Keywords: mechanical systems, vibration analysis, computational mechanics, accelerometers

\section{INTRODUCTION}

The Nowadays, many industrial plants require a section dedicated to air treatment, also in large installations addressed to the production of electrical energy. One of the tasks of this subsystems is to regulate the airflow rate at the inlet to maintain a desired value, preventing overpressures inside ducts. To achieve this need, a gravity damper is usually adopted; it is a unidirectional valve diverting the undesired excess of air mass from the flow through its opening under conditions of overpressure. If no overpressure conditions are acting on the damper, it remains in its normal state: closed under the action of gravity force. This device can however be used also as a direct restrictor valve on the main inlet duct. The common architecture of a gravity damper consists of a rectangular shaped frame (depending on the dimensions of the duct), housing a variable number of dimensionally equal sections (Figure 1).

The present work deals with a gravity damper whose sections are constituted by rectangular AISI sheets: by means of bolts and nut connections, they are rigidly coupled with a shaft of squared cylinder shape. This kind of valves can face severe working conditions, especially for applications related to energy conversion systems as gas turbines, because of the large amounts of treated air flow speeds. In particular, dynamic phenomena can lead to structural failures, highly undesired in this kind of plants. The prevention of these dangerous phenomena is so important that the UNI EN 1751:2003 suggests a very cautionary test for this kind of device, with a flow speed of $150 \%$ with respect to the nominal value. By a structural point of view, vortex shedding [1] [2] is the main dynamic phenomenon to keep an eye on; it consists in the alternate detachment of vortexes from the upper and lower faces of a section with a certain frequency, depending on its shape and flow speed, hence on the Reynolds number. This behaviour is present in subsonic conditions even if the flow has constant speed and direction. The detachment generates forces acting on the structure and, when the frequency is in proximity to the resonance frequency of the body, the lock-in phenomenon [3, 4] can occur, leading to the amplification of oscillations.

Since the lock-in is generated by a very unsteady phenomenon, its comprehension is challenging by the point of view of theoretical modelling, numerical simulations, experimental analysis and has therefore stimulated a certain interest in the scientific literature. For completeness, a comprehensive classification of 
possible vortex induced vibration (VIV) models is presented in [4].

The present work, as shall be explained in the following, stands at the crossroad between numerical simulation and experimental analysis of VIV and its motivations are actually based on the state of the art in the literature about these two approaches.

As regards numerical modelling, the main issues are computational cost and unsteadiness. At low Reynolds numbers it is still possible [5] [6] to capture the main physics of VIV (for relatively simple systems as circular cylinders) using the Reynolds-averaged Navier-Stokes (RANS) formulation. In particular, in [6] it is discussed how the RANS formulation smooths the randomness in an intelligible way and therefore, comparing numerical predictions against experimental measurements, some random characteristics of the VIV response can be revealed. In [7], the VIV response of four circular cylinders in a square configuration is studied through the RANS approach. Although, as discussed above, the RANS turbulence models can be effective for the comprehension of VIV, the turbulence Large-Eddy Simulation (LES) models are required every time that the computational investigation is focused on the detachment phenomena at high frequency, as is the case of VIV at high or even moderate Reynolds number. In [8], the vortex shedding phenomenon is studied for a squared cylinder with low Reynolds number by means of unsteady RANS (URANS) and LES simulations, comparing the numerical results with experimental ones. The main conclusion is that the URANS model doesn't reproduce correctly the vortex shedding, on the contrary of the LES simulation that instead has the drawback of an impressive computational cost. A milestone as regards the comparison of the performances of RANS and LES approaches at high Reynolds number is [9]: vortex-shedding flow past a square cylinder at $\mathrm{Re}=22.000$ and of the $3 \mathrm{D}$ flow past a surface-mounted cube at $\mathrm{Re}=40.000$ are studied. In [10], the accuracy of LES with wall modelling for high Reynolds number complex turbulent flows is investigated by considering the flow around a circular cylinder in the supercritical regime. Particular attention is devoted to how the mismatch between experimental data and LES and RANS simulation results evolves with increasing Reynolds number. In [11], LES is adopted for studying the vortex shedding from a square cylinder at high Reynolds number and a filtering procedure is introduced in the frequency space to separate the periodic from the turbulent fluctuations. In [12], the very high Reynolds number regime (order of $10^{6}$ ) is investigated. On these grounds, for the present work a Quasi-2D LES turbulence model is adopted. The LES model has been considered necessary for the desired level of comprehension of vortexes on the selected test case gravity damper having Reynolds number of the order of $10^{5}$. Computational cost has been saved because it is possible to reduce the three-dimensional structure of the gravity damper of Figure 1 to three twodimensional design configurations: without bolts and nuts, with bolt facing forward, with bolt turned back (Figures 2 and 3). The main pro of this approach is that it allows to spread in only two dimensions a very fine mesh and therefore to estimate if and how the presence of small elements like bolts affects the vortex shedding.

As regards experimental techniques for the detection of vortex shedding, the possible approaches are several: for example, flow visualization and hot-wire measurements are often used to get a qualitative and quantitative assessment of the evolution of the flow [13]. Accelerometric acquisition is a very powerful approach for the detection of vortex shedding and its versatility allows employing it on a variety of systems. In [14] and [15], the method was adopted for detecting vortex shedding on a large bridge; in [16] the oscillations generated by the action of the wind on a wind turbine tower were monitored through 15 accelerometers; in [17], the vortex shedding on long flexible cylinders (e.g., risers, tendons and mooring lines) exposed to ocean currents is studied by exploiting data from strain gauges and accelerometers. The data are used to model the fatigue damage of the structures. Long flexible pipelines are investigated also in [18], through an ad hoc designed and built experimental facility: it consists of direct local fluid force measurement at two locations on the cylinder as well as accelerometers spaced along the cylinder axis.

The main feature of the experimental analysis above cited is that they are remote conducted or (as in the case of [19]) they are conducted on a smallscale model of the real system of interest. In virtue of the dimensions of the test case gravity damper, this work instead deals with a full-scale experimental campaign conducted at the wind tunnel facility of the University of Perugia. This allows precision control on the experimental conditions and, most of all, to collect accelerations (as done also in [20]) occurring during hardest working condition, whose time-history could be useful for structure fatigue analysis.

Summarizing, the objective of this study is the following: the interpretation of VIV on one closure section of the test gravity damper, through the CFD numerical model, and consequently its experimental detection. On the basis of the indications from the numerical model, finally, it is possible to formulate some strategies for mitigating VIV and the availability of the full-scale system at the wind 
tunnel facility allows to really test these strategies and evaluate them.

The structure of the Paper is therefore as follows: Section 2 is devoted to the statement of the objectives and to the description of the set up of the CFD model of the gravity damper. Section 3 is devoted to the description of the experimental set up. In Section 4, the numerical and experimental results are collected and the mitigation strategy of VIV is presented and discussed. In Section 5, finally, the conclusions are drawn and some further directions of the present work are indicated.

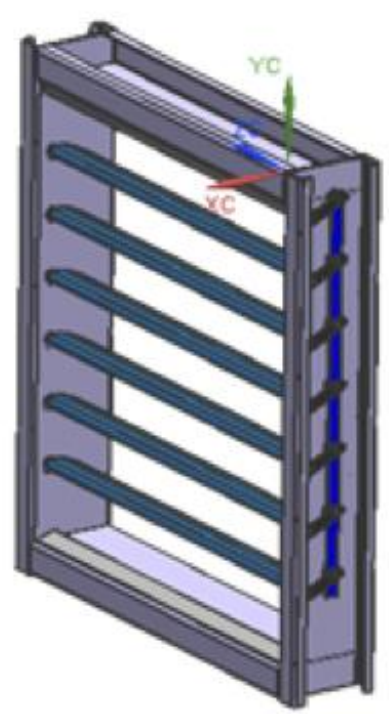

Fig. 1. CAD model of one of the analysed gravity damper

\section{NUMERICAL ANALYSIS}

Since the structure of the system must be safe and since in the literature full-scale tests for similar systems are uncommon, it is extremely useful to obtain information on the aerodynamic conditions of the flow in proximity of the most stressing working conditions through numerical modelling. For this reason, CFD simulations at $150 \%$ of the nominal wind speed have been conducted. The Reynolds number of the test system, calculated using the section length, is $4.6 \cdot 10^{5}$ and, at these scales, the energy content of a wake of vortexes is surely considerable. As reviewed in the Introduction, VIV at high Reynolds numbers has been investigated in the literature through several theoretical approaches for simple structures. When the geometry of the section is complex, it is difficult to find adequate theoretical models [4] and, further, in the case of the selected gravity damper (Figure 1), three different sections can be identified and should be studied.

For these reasons, a numerical CFD simulation is fundamental in order to understand the phenomenology of VIV on the system of interest. A Quasi-2D CFD analysis has been selected, considering separately the three main sections of the system. Actually, spreading the computation mesh on the full three-dimensionality of the object, the results wouldn't have the desired precision and coherence. This choice allows adopting a LES turbulence model, which is more appropriate for highly unsteady phenomena as VIV at a Reynolds number of the order of $10^{5}$. The analysis has been performed using the code Star-ccm+ by CDAdapco. The main mesh and physical parameters selected for the simulations are summarized in Tables 1 and 2 .

Table 1. Main parameters of the mesh

\begin{tabular}{|l|c|}
\hline Type & $\begin{array}{l}\text { Polyhedral mesh with } \\
\text { prism layer }\end{array}$ \\
\hline $\begin{array}{l}\text { Minimum and } \\
\text { maximum values }\end{array}$ & $0.5-20 \mathrm{~mm}$ \\
\hline $\begin{array}{l}\text { Number of wall prism } \\
\text { layer }\end{array}$ & 10 \\
\hline Prism layer thickness & 1 \\
\hline Total number of cells & 1674659 \\
\hline
\end{tabular}

Table 2. Main parameters of the physical model

\begin{tabular}{|l|c|}
\hline Type & Quasi - 2D Unsteady \\
\hline Turbulent model & LES \\
\hline Time step value & $10^{-4} \mathrm{~s}$ \\
\hline $\begin{array}{l}\text { Velocity inlet Boundary } \\
\text { Condition }\end{array}$ & $45 \mathrm{~m} / \mathrm{s}$ \\
\hline
\end{tabular}

LES is a mathematical model [21] [22] used in Computational Fluid dynamics (CFD) for the study of highly turbulent phenomena. It stands as a compromise between a Reynolds averaged NavierStokes (RANS) modelling (faster but more approximate) and a direct numerical simulation (DNS, more accurate but prohibitive in terms of computational time). The LES model performs an explicit solution of large eddies and implicitly accounts for the small eddies by using a subgridscale model (SGS model). In this case, to evaluate the eddy viscosity, the wall-adapting local eddyviscosity (WALE) model by Nicoud and Ducros [23] was adopted. Instead of considering the device as a whole, three different configurations were analysed: without bolt and nut, bolt facing forward, bolt turned back (Figure 2). This was done in order to understand the VIV contribution in each case (Figure 3).

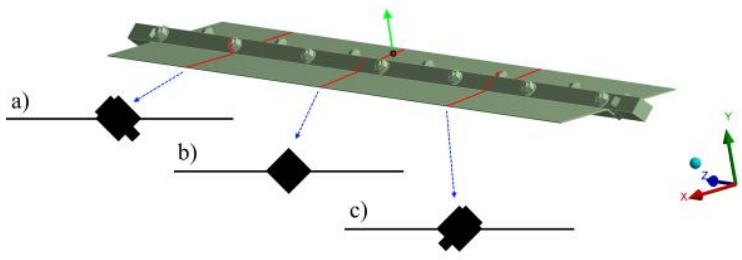

Fig. 2. The system and the three sections analysed. The dimensions of the system are $155 \times 988 \times 1.5$ $\mathrm{mm}$. The point and the arrow indicate respectively the position and the measuring direction of the accelerometer 


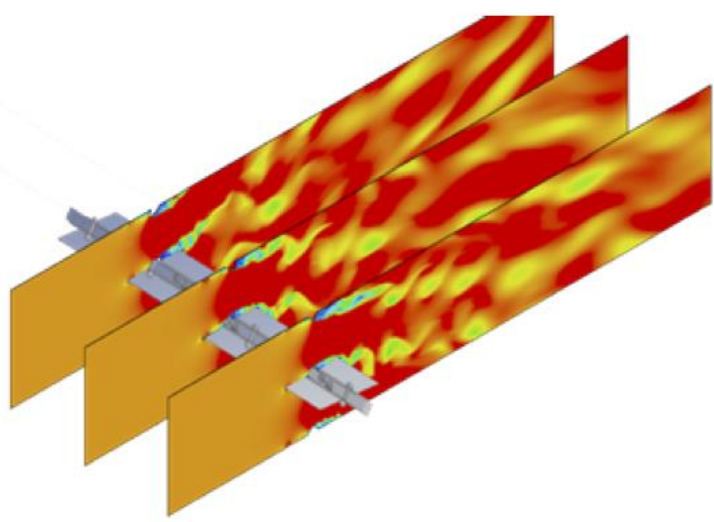

Fig. 3. Sections analysed through the CFD model

A modal analysis was performed to identify both the modal shapes and the natural frequencies of the system. The model of the structure was implemented at full opening. All the components were modelled in AISI 316L. The sheet metal part of the wing was implemented as a surface model (SHELL 181 structural element of the ANSYS library), while for the axle, bolts and nuts, SOLID187 elements were chosen. Fixed support was considered near the ends of the axis. The model is thus made of 25967 elements and 6336 nodes. The first ten vibration frequencies, associated to their modal participation factors and expressed in the percentage that compels it for the composition of the modal form, are reported in Figure 4. Excluding the first and the third mode, associated to the bending axis on mutually perpendicular planes, all the other vibration modes relate to the fluttering of the metal sheet [24]; increasing frequency corresponds to increasing number of nodes. The second mode of vibration regards the bending to 3 nodes of the axis and also involves the sheet. As an example, the 4th and 6th modal shapes are reported in Figure 5. On the grounds of the set up of the system and of the accelerometer position (Figure 2), it is expected that most relevant frequencies should be $241 \mathrm{~Hz}$ (Rot Z) and $304 \mathrm{~Hz}$ (Y oscillations).

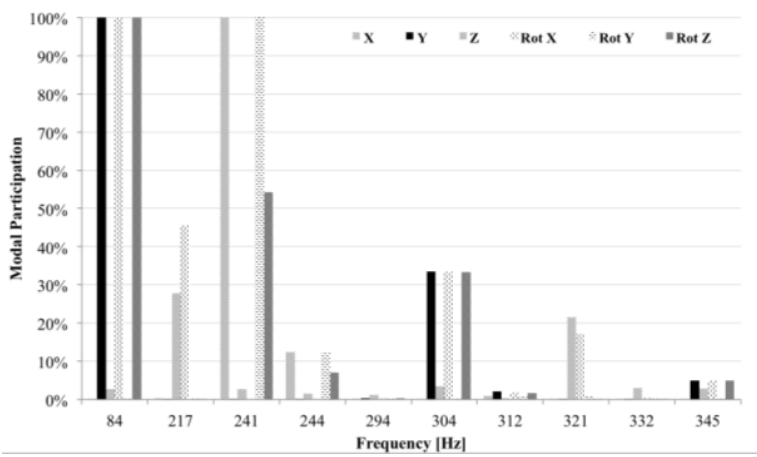

Fig. 4. Numerical natural frequencies, with their modal participation. For the orientation of the axis, refer to Figure 2 a)

b)
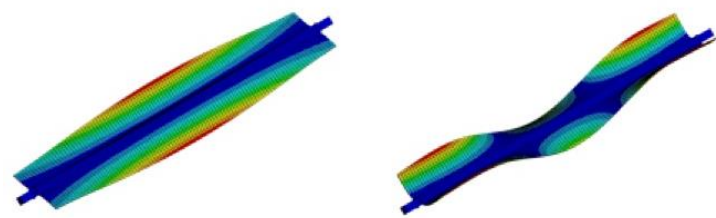

Fig. 5. 4th modal shape, $244 \mathrm{~Hz}$ (a), 6th modal shape, $304 \mathrm{~Hz}$ (b)

\section{THE EXPERIMENTAL SET UP}

A full-scale wind tunnel investigation was carried out at the "Raffaele Balli" wind tunnel facility of the University of Perugia (www.windtunnel.unipg.it). The objective of the investigation was to detect and evaluate the VIV on the device. The wind tunnel is in a closed loop configuration. A fan, driven by a $375 \mathrm{~kW}$ electric motor, with 11 blades in carbon warped shell, accelerates the air up to a maximum speed of 50 $\mathrm{m} / \mathrm{s}$. In each curve, there are fixed blades to enhance the air flow pattern. Just prior to the test room (approximately $4 \mathrm{~m}^{2}$ of effective section), the turbulence of the flow is conditioned and reduced by means of a honeycomb section. The flow is further accelerated by a converging duct into the testing room. Both the static and dynamic air conditions are monitored and correlated to the aerodynamic forces. Two Pitot tubes measure the wind speed blowing on the test section and a control station in the testing room records in real time data about static air pressure, temperature and relative humidity.

The gravity damper analysed in this work is composed of four full-scale closure sections (Figure $6)$.

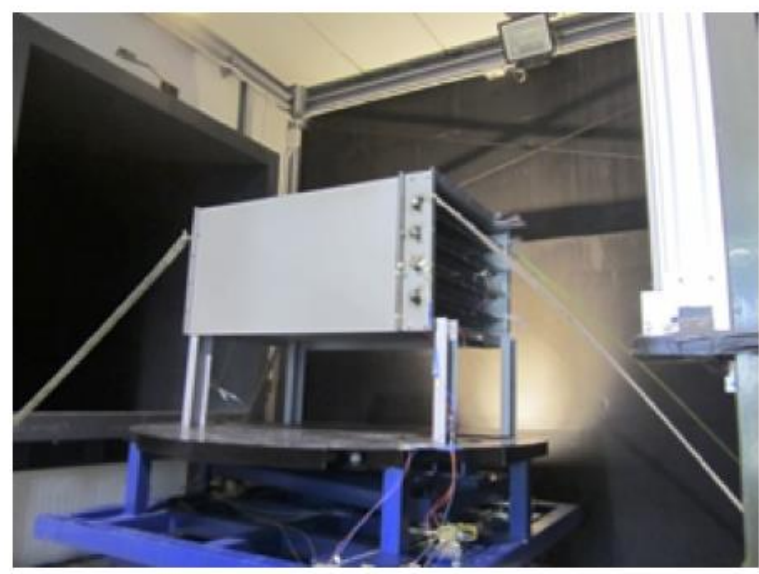

Fig. 6. The gravity damper during a test

The body to test was placed on the aerodynamic scales, respecting its opening orientation. The tests were divided in three steps:

1. Rising speed ramp;

2. Regime;

3. Decreasing speed ramp. 
The tests were carried out at a regime speed intensity of $45 \mathrm{~m} / \mathrm{s}$ and were thrice replicated. The blockage ratio is $15 \%$ when the gravity damper is closed but, at around $20 \mathrm{~m} / \mathrm{s}$ of wind speed, the device is already completely open and the blockage ratio drops to $4 \%$. The gravity damper was instrumented with 5 miniaturized accelerometers each weighing 2.3 grams; one of them was placed in the middle of the section (Figure 2). Signals were acquired at $5 \mathrm{kHz}$ and then low pass filtered at 2 $\mathrm{kHz}$. A Prosig P8000 data logger was used to collect data concerning accelerations on the structure, wind speed and opening percentage.

\section{THE RESULTS}

The main objective of the numerical analysis was to highlight phenomena of detachment from the fluid vein that, in terms of floating pressures acting on the structure, might trigger undesired large vibrations. The maximum of the Q-criterion [25] is a reliable method for locating vortexes and, for this reason, the vorticity contour plot and the QCriterion contour plot are adopted. In Figure 7 and Figure 8, respectively, the vorticity contour plot and the Q-Criterion contour plot for the case without bolt and nut are reported. It has been observed that, for each of the three analysed sections of the system, similar alternate vortexes detachment in the wake of the component is predicted.

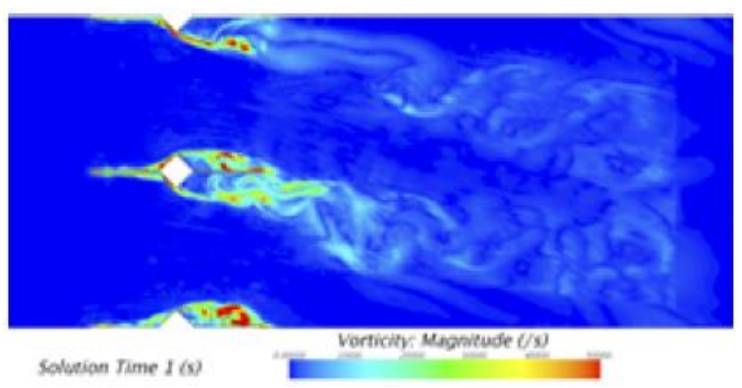

Fig. 7. Vorticity contour plot of the section without bolt and nut

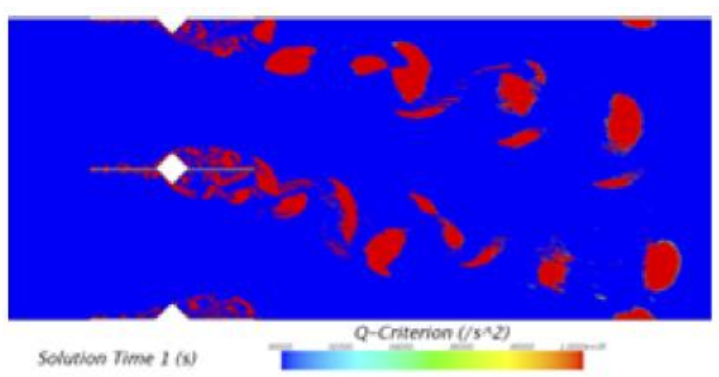

Fig. 8. Q-Criterion contour plot of the section without bolt and nut

The Q-Criterion locates regions where rotation dominates the strain in the flow: $Q$ is defined as the difference between the modulus squared of the spin tensor and the modulus squared of the strain rate tensor. When $\mathrm{Q}$ is positive, the flow is vorticity- dominated; a negative value corresponds instead to strain-dominated flow. It is easy to individuate the formation of the vortexes around the object and their evolution into the control volume. In particular, for the tested gravity damper, it arises that the propagation of vortexes after their detachment from the wake is sharp.

Since the Q-criterion, for all the three analysed sections, has highlighted aerodynamic fields dominated by alternate vortexes in the wake of the component under analysis, the frequency contributions that these pressure fields impose have been interpreted by comparing the predictions from the numerical models to the measured acceleration signal. In Figure 9, the Power Spectral Density (PSD) is shown of the measured vibration signal and of the lift coefficients predicted by the CFD model for the three sections of interest (Figure 2). The spectra are normalized to a reference value: for each case, it is the amplitude at $10 \mathrm{~Hz}$. From Figure 9 , it arises that, as expected, the experimental spectrum has a more complex shape with respect to the numerical spectra: actually, the system is a complex structure and consequently its spectrum resembles the richness of structural and fluid dynamics vibration modes. For the objectives of this study, the area circled in Figure 9 (corresponding more or less to the $[220,320] \mathrm{Hz}$ interval) is particularly relevant as regards the comparison between simulations and experiment: on the numerical side, the vortex shedding frequency is clearly visible; on the experimental side, a more complex pattern of peaks is present. Recall that a vortex shedding frequency of the order of $270 \mathrm{~Hz}$ is plausible for the system of interest, as suggested in [26] where the shedding frequency is computed of a square cylinder ( $200 \mathrm{~mm}$ each side) with $45^{\circ}$ of incidence at a Reynolds number of $5.5 \cdot 10^{4}$. These results motivate a deeper investigation of the experimental signal and this is done by windowing it through the Welch filter technique [27] for a transient analysis aimed at studying the evolution of the most energetic frequencies. In Figure 10, the spectrum over three different windows of 1 second is shown. The first refers to the very incipient phase of blowing wind and therefore the structural response is the only visible (peak around $300 \mathrm{~Hz}$, predicted also by the modal analysis of Figure 4). The second window refers to the regime of wind rising ramp, approaching steady wind, and therefore the complex structure of vortexes and the complex physics of structural vibrations reflects in a variegated pattern of peaks in the spectrum. The third window refers to the steady wind speed regime. As in Figure 10 is clearly visible, at regime there is a slight tendency towards the lock in because the structural peak moves downward and there is another peak at a frequency typical of vortex shedding. 


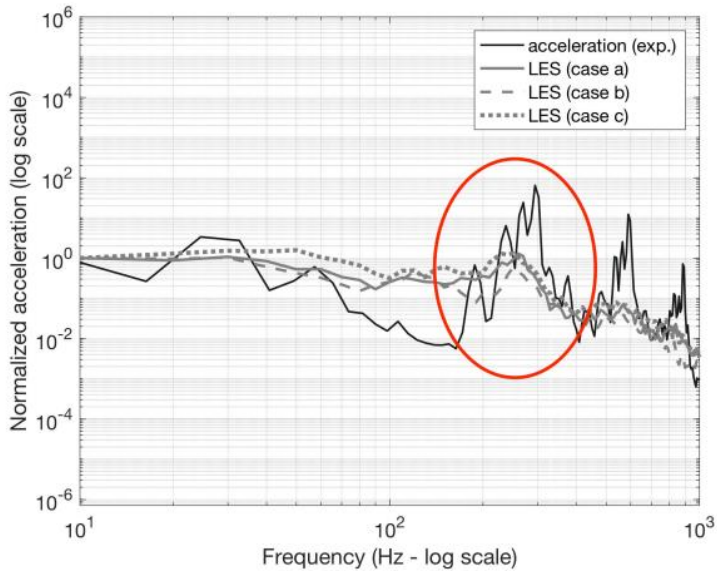

Fig, 9. Power spectral density (PSD) of the experimental acceleration and of the numerical lift coefficients. The three cases refer to the sections of Figure 2. The amplitudes are normalized to the reference value at $10 \mathrm{~Hz}$

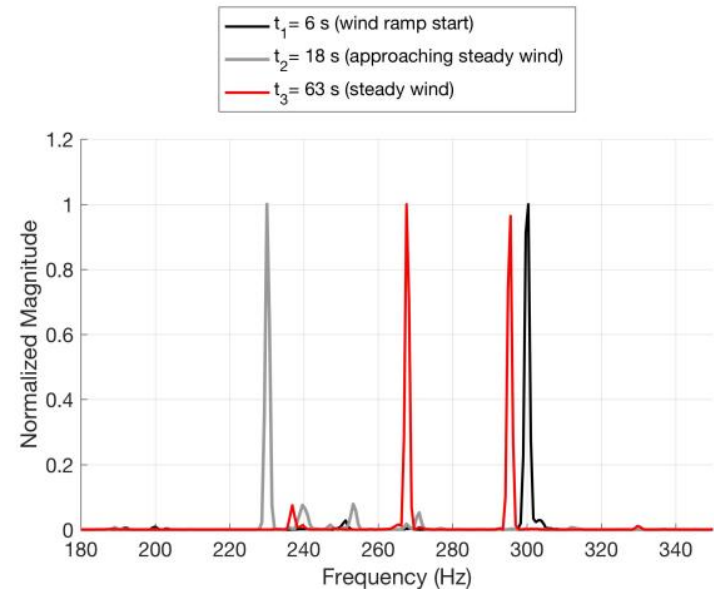

Fig. 10. Power spectral density (PSD) of Welch filtered windows of the experimental acceleration

The frequency content of the accelerometric acquisition is analysed also through the continuous wavelet transform (Figure 11), synchronized with the air speed of the wind tunnel. This kind of representation is very useful to individuate the composition of the frequency content of the signal for experimental tests with transient and unsteady phenomena [28]. The resulting picture is compatible with what arises in more detail from Figure 10. The Welch filter is actually more capable of capturing the transient and at the same time highlighting the most relevant peaks.

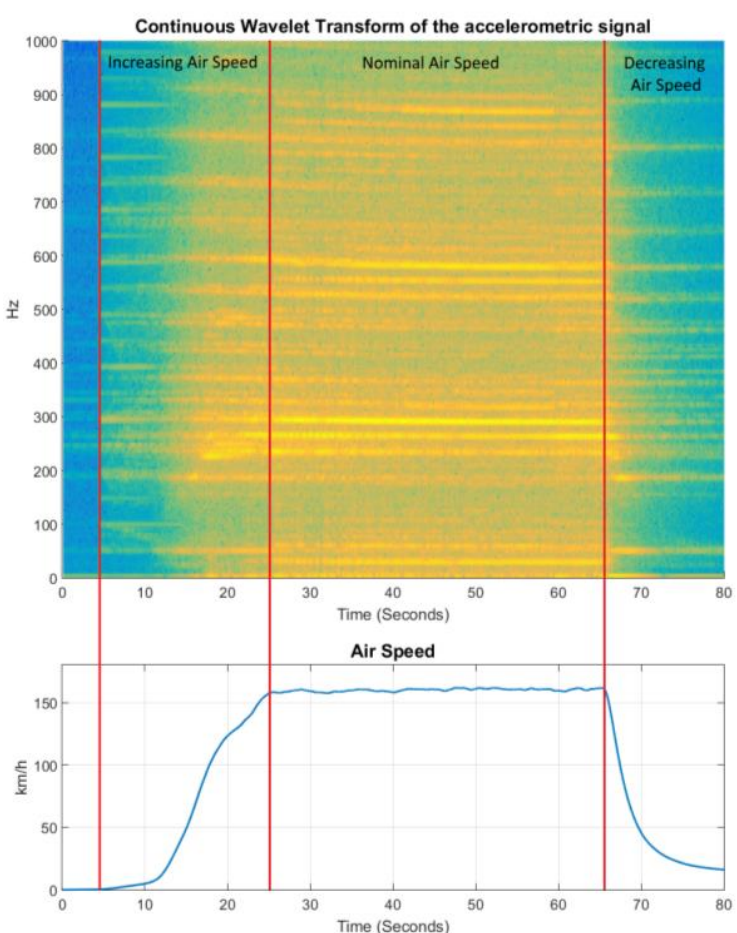

Fig. 11. Wavelet Continuous Transform of the acceleration time series measured in the wind tunnel

Finally, the strategies for mitigating the VIV are addressed. To give an order of magnitude of the diagnosed vibration issue, the measured acceleration time series is reported in Figure 12.

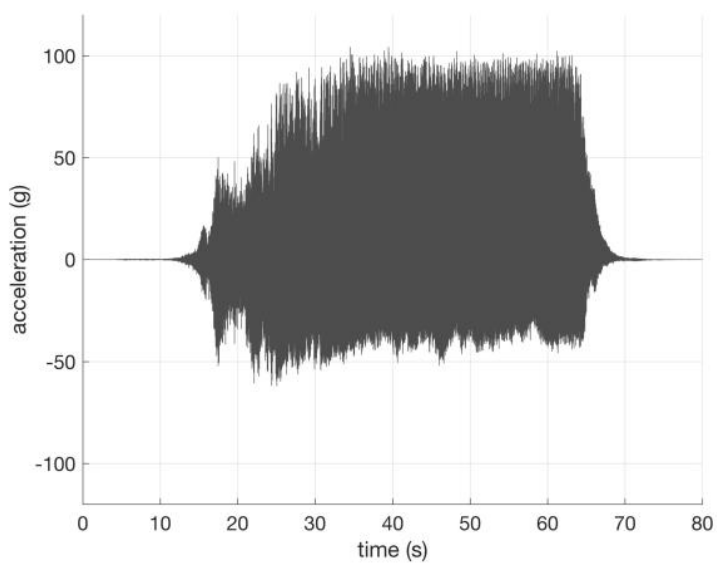

Fig. 12. Time series of the measured acceleration of the device during the test in the wind tunnel

On the grounds of these numerical and experimental results, the strategies for mitigating the amplitude of VIV can be analysed. Basing on the CFD model (Figure 7 and Figure 8) and in particular on the prediction that the presence and the orientation of bolts and nuts doesn't affect vortices detachment, it is conceivable to mitigate VIV only by acting on the closure section. Two different solutions have actually been experimented in the wind tunnel. In the former case, the idea is acting directly on the vortex shedding frequency, in 
the latter case the natural frequencies of the structure are moved. So, in the first case, an airfoil profile was substituted to the actual closure section and in the second case the cross section of the whole device was maintained, but the longitudinal dimension of the closure section was halved. The graph in Figure 13 shows the comparison between the Root Mean Square (RMS) of the acceleration signal at the middle of the closure section, tested and averaged in a second of acquisition for the three cases tested in the wind tunnel. The red line refers to the system as it is, the blue and the black lines correspond to the two mitigation strategies. From Figure 13 it arises that both strategies actually provide a severe mitigation of VIV, of the order of 15 times averagely. In particular, the airfoil profile strategy leads to a slightly better mitigation.

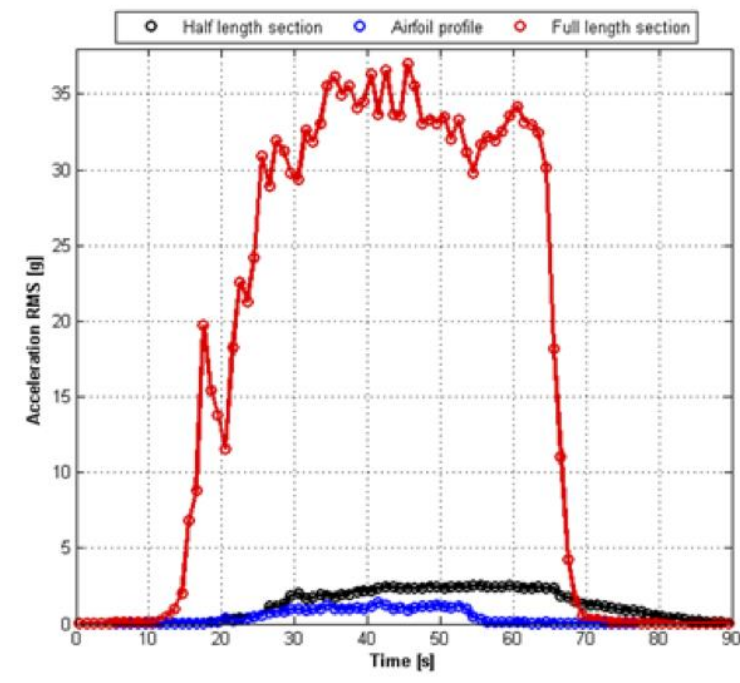

Fig. 13. RMS averaged in one second of acquisition for the system as it is (red line) and for the two strategies of VIV mitigation (blue and black lines)

\section{CONCLUSIONS}

Gravity dampers are one-way valves, normally closed under the effect of gravity. They are widely used in the air treatment ducts. If the installation has to process large masses of air at high speed, as is the case of energy conversion systems (for example, inlet turbine engines pipes), an accurate study of the shape and dimensions of the closure section should be conducted. The vortex-induced vibration can, in fact, trigger some disadvantageous dynamic effect. Not only this: if the shedding frequency and some natural frequency of the structure are close, the aeroelastic lock-in can occur, drastically reducing the life of the component. On these grounds, in this study, VIV on a test case gravity damper has been diagnosed experimentally, interpreted through numerical modelling and has been mitigated through design improvement on the test device. The main results confirm that the common industrial design processes actually lack the scientific accuracy needed for understanding and handling very unsteady phenomena on complex systems at relatively high Reynolds numbers, as is the case for the selected system. The numerical simulations, in the form of a Quasi-2D LES CFD model and a modal analysis, resulted fundamental in order to characterize vortex detachment and in order to interpret the observed frequency contributions to VIV. A full-scale wind tunnel campaign, with an accelerometer-based vibration analysis, has been conducted too and it has therefore been possible to compare the frequency content of the experimental and simulated data. The CFD model allowed interpreting the large amplitude of VIV as due to a tendency towards the lock-in because of the approaching of the vortex shedding frequency (Figure 9) to a natural frequency of the system (Figure 10). The CFD analysis has been conducted on three different sections of the system, in order to appreciate if the presence and the orientation of bolts and nuts considerably affect VIV. This not being the case, these results from the CFD model inspire possible strategies of VIV mitigation: the main contribution to VIV is due to the shape and the orientation of the shaft and the intervention should be and has actually been, in this work, focused on this aspect. Having at hand the full-scale system, to be tested experimentally in the wind tunnel, two different configurations of the closure section have actually been designed, realized and tested with the objective of avoiding the onset of large amplitude VIV, by creating a gap between the natural frequencies of the structure and those of the vortex shedding. Both mitigation strategies actually provide a drastic reduction in the amplitude of VIV: this has been crosschecked by measuring the RMS of the acceleration signal at the closure section.

Summarizing, in this study the need has been highlighted for a comprehensive scientific approach for the characterization of systems and processes, that are very common in the industry: the reason for this need is that they likely involve highly unsteady phenomena triggering non-trivial dynamic effects. The results of this work point out that an integrated approach and the interplay of numerical modelling and experimental vibration analysis are very powerful for interpreting the dynamic behaviour of systems having complex shapes, as the selected test case gravity damper is. Moreover, the identification of strategies for mitigating VIV on the selected test case highlights that such an integrated approach is particularly valuable in the early design phase of the systems. Further, the accelerogram produced as one of the results of this work could be inspiring for the study of the fatigue damage of the structure.

\section{REFERENCES}

1. Bearman PW. Vortex shedding from oscillating bluff bodies. Annual review of fluid mechanics, 
1984, 16(1):195-222.

https://doi.org/10.1146/annurev.fl.16.010184.001211

2. Griffin O, Skop R, Koopmann G. The vortex-excited resonant vibrations of circular cylinders. Journal of Sound and Vibration, 1973, 31(2):235-249. https://doi.org/10.1016/S0022-460X(73)80377-3

3. Blackburn $\mathrm{H}$, Henderson R. Lock-in behavior in simulated vortex-induced vibration. Experimental Thermal and Fluid Science, 1996; 12(2):184-189. https://doi.org/10.1016/0894-1777(95)00093-3

4. Paidoussis MP, Price SJ, De Langre E. Fluidstructure interactions: cross-flow-induced instabilities. Cambridge University Press 2010.

5. Constantinides Y, Oakley $\mathrm{OH}$, Numerical prediction of bare and straked cylinder viv. In 25th International Conference on Offshore Mechanics and Arctic Engineering, American Society of Mechanical Engineers; 2006:745-753. https://doi.org/10.1115/OMAE2006-92334

6. Pan Z, Cui W, Miao Q. 2007. Numerical simulation of vortex-induced vibration of a circular cylinder at low mass-damping using rans code. Journal of Fluids and Structures, 2007;23(1):23-37.

https://doi.org/10.1016/j.jfluidstructs.2006.07.007

7. Zhao M, Cheng L. Numerical simulation of vortexinduced vibration of four circular cylinders in a square configuration. Journal of Fluids and Structures, 2012; 31: 125-140.

https://doi.org/10.1016/j.jfluidstructs.2012.04.002

8. Murakami S, Mochida A. On turbulent vortex shedding flow past $2 \mathrm{~d}$ square cylinder predicted by cfd. Journal of Wind Engineering and Industrial Aerodynamics, 1995; 54:191-211. https://doi.org/10.1016/0167-6105(94)00043-D

9. Rodi W. Comparison of les and rans calculations of the flow around bluff bodies. Journal of wind engineering and industrial aerodynamics, 1997;69: 55-75. https://doi.org/10.1016/S01676105(97)00147-5

10. Catalano P, Wang M, Iaccarino G, Moin P. Numerical simulation of the flow around a circular cylinder at high reynolds numbers. International Journal of Heat and Fluid Flow, 2003;24(4):463-469. https://doi.org/10.1016/S0142-727X(03)00061-4

11. Bouris D, Bergeles G. 2d les of vortex shedding from a square cylinder. Journal of Wind Engineering and Industrial Aerodynamics, 1999;80(1):31-46. https://doi.org/10.1016/S0167-6105(98)00200-1

12. Ong MC, Utnes T, Holmedal LE, Myrhaug D, Pettersen B. Numerical simulation of flow around a smooth circular cylinder at very high reynolds numbers. Marine Structures, 2009;22(2):142-153. https://doi.org/10.1016/j.marstruc.2008.09.001

13. Segalini A, Talamelli A. Experimental analysis of dominant instabilities in coaxial jets". Physics of fluids, $201123(2)$ : 024103

https://doi.org/10.1063/1.3553280

14. Flamand O, De Oliveira F, Stathopoulos-Vlamis A, Papanikolas P. Conditions for occurrence of vortex shedding on a large cable stayed bridge: Full scale data from monitoring system. Journal of Wind Engineering and Industrial Aerodynamics, 2014; 135: 163-169. https://doi.org/10.1016/j.jweia.2014.07.011

15. Li H, Laima S, Ou J, Zhao X, Zhou W, Yu Y, Li N, Liu Z. Investigation of vortex-induced vibration of a suspension bridge with two separated steel box girders based on field measurements. Engineering Structures, 2011;33(6):1894-1907. https://doi.org/10.1016/j.engstruct.2011.02.017
16. Molinari M, Pozzi M, Zonta D, Battisti L. Infield testing of a steel wind turbine tower. In Structural Dynamics and Renewable Energy, Volume 1. Springer, 2011: 103-112.

17. Mukundan H, Modarres-Sadeghi Y, Dahl JM, Hover FS, Triantafyllou MS. Monitoring viv fatigue damage on marine risers. Journal of Fluids and structures, 2009;25(4): 617-628. https://doi.org/10.1016/j.jfluidstructs.2009.03.0 $\underline{03}$

18. Marcollo H, Hinwood J. On shear flow single mode lock-in with both cross-flow and in-line lock-in mechanisms. Journal of Fluids and structures 2006;22(2): 197-211. https://doi.org/10.1016/j.jfluidstructs.2005.10.0 01

19. Diana G, Fiammenghi G, Belloli M, Rocchi, D. Wind tunnel tests and numerical approach for long span bridges: the messina bridge. Journal of Wind Engineering and Industrial Aerodynamics, 2013; 122: 38-49. https://doi.org/10.1016/j.jweia.2013.07.012

20. Scappaticci L, Mariani F, Bartolini N, Risi F, Garinei A. Dynamic effects of wind loads on a gravity damper. Procedia Engineering, 2015;109:162-170. https://doi.org/10.1016/j.proeng.2015.06.228

21. Kleissl J. Field experimental study of the Smagorinsky model and application to large eddy simulation. 2004.

22. Kobayashi $T$. Large eddy simulation for engineering applications. Fluid dynamics research, 2006;38(2):84-107. https://doi.org/10.1016/j.fluiddyn.2005.06.004

23. Nicoud F, Ducros F. Subgrid-scale stress modelling based on the square of the velocity gradient tensor. Flow, turbulence and Combustion, 1999;62(3): 183-200. https://doi/org/10.1023/A:1009995426001

24. Dowell EH. Nonlinear oscillations of a fluttering plate. AIAA journal, 1966;4(7): 1267-1275. https://doi.org/10.2514/3.3658

25. Hunt JC, Wray AA, Moin P. Eddies, streams, and convergence zones in turbulent flows. In Studying Turbulence Using Numerical Simulation Databases, 2. Proceedings of the 1988 Summer Program, Stanford University, pp. 193-208.

26. Mueller AA. Large Eddy Simulation of crossflow around a square rod at incidence with application to tonal noise prediction. University of Twente. 2012.

27. Welch P. The use of fast fourier transform for the estimation of power spectra: a method based on time averaging over short, modified periodograms. IEEE Transactions on audio and electroacoustics, 1967;15(2): 70-73. https://doi.org/10.1109/TAU.1967.1161901

28. Laurantzon F, Örlü R, Segalini A, Alfredsson PH. Time-resolved measurements with a vortex flowmeter in a pulsating turbulent flow using wavelet analysis. Measurement Science and Technology, 2010; 21(12), 123001. https://doi.org/10.1088/0957$0233 / 21 / 12 / 123001$

Received 2018-01-08

Accepted 2018-03-09

Available online 2018-03-12 


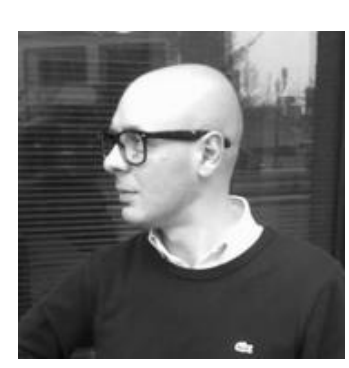

Lorenzo SCAPPATICCI is a research fellow at Guglielmo Marconi University, Rome. His research interests deal mainly with Finite Element Methods for structural optimization of vehicles and for bioengineering applications; with the analysis of fluid -structures interactions; with vibration analysis and wind tunnel testing of vehicles.

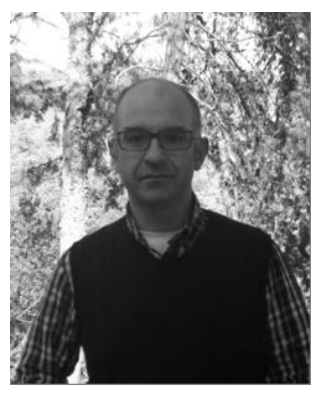

Francesco CASTELLANI is an Associate Professor in Machine Engineering teaching Applied Mechanics at the University of Perugia, Italy. $\mathrm{He}$ is involved in many research activities dealing with modelling and control of mechanical systems. His research is focused on numerical and experimental wind turbines studies, wind resource assessment, numerical simulation of wind flow and wakes on complex terrain sites, condition monitoring and fault diagnosis through SCADA data analysis and wind tunnel test of micro wind turbines.

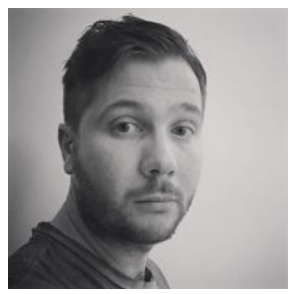

Davide ASTOLFI is a post-doc at the Department of Engineering of University of Perugia, Italy. His research activities deal mainly with modelling and control of mechanical systems, condition monitoring and fault diagnosis of wind turbines through data mining and numerical simulation of wind flow and wakes. He also works on wind tunnel testing of micro wind turbines and vehicles for aerodynamic and performance analysis.

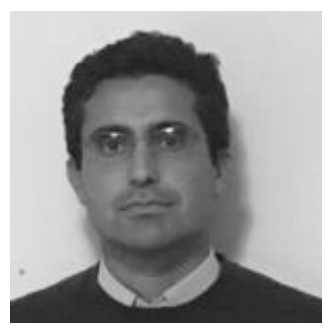

Alberto GARINEI is an Associate Professor in Mechanical Measurements at Guglielmo Marconi University, Rome. 